\title{
Efectos intergranulares en perovskitas de manganeso nanocristalinas
}

\author{
L.E. HUESO ${ }^{1}$, A. FONDADO ${ }^{1}$ Y J. RIVAS ${ }^{1}$, F. RIVADULLA ${ }^{2}$, M.A. LÓPEZ-QUINTELA ${ }^{2}$ \\ ${ }^{1}$ Departamento de Física Aplicada, Universidad de Santiago de Compostela, 15706 Santiago de Compostela, España. \\ ${ }^{2}$ Departamento de Química-Física, Universidad de Santiago de Compostela, 15706 Santiago de Compostela, España.
}

\begin{abstract}
En este artículo se examinan los efectos intergranulares sobre el transporte eléctrico en muestras cerámicas de perovskitas de manganeso que presentan magnetorresistencia colosal. La progresiva reducción del tamaño de partícula que nos permite la tecnología sol-gel hace que estos materiales muestren nuevas e inesperadas propiedades. Así, en la zona de la transición metal-aislante, se destruye la magnetorresistencia intrínseca del compuesto para tamaños de partícula $\mathrm{D} \leq 150 \mathrm{~nm}$. A bajas temperaturas $(\mathrm{T}<30 \mathrm{~K})$, la aparición de un comportamiento activado de la resistividad sugiere la presencia de Bloqueo de Coulomb entre granos.
\end{abstract}

Palabras clave: magnetorresistencia, manganitas, propiedades eléctricas.

\section{Intergranular effects on nanocrystalline manganese perovskites}

Intergranular magnetotransport effects are studied on polycrystalline manganites. Reducing grain size through sol-gel technology allows us to show new results on these materials. Intrinsic colossal magnetoresistance is destroyed in metalinsulator phase transition for grain size smaller than $150 \mathrm{~nm}$. In low temperature region, that is, $\mathrm{T}<30 \mathrm{~K}$, semiconductor resistivity behavior indicates Coulomb blockade between grains.

Keywords: magnetoresistance, manganites, electrical properties.

\section{INTRODUCCIÓN}

Los fenómenos de magnetorresistencia (MR) han sido objeto de un estudio intensivo desde el descubrimiento de la magnetorresistencia gigante hace ya 10 años en multicapas de $\mathrm{Fe} / \mathrm{Cr}$ (1) o en aleaciones granulares (2). Más recientemente, la aparición de altísimos valores de magnetorresistencia en perovskitas de manganeso con valencia mixta (la después llamada magnetorresistencia colosal (MRC)) $(3,4)$ ha provocado que este tipo de materiales pase a primer plano en la investigación científica, tanto desde el punto de vista teórico como desde el experimental. La posible aplicación de estos fenómenos en la industria de la electrónica ha sido uno de las causas que justifican el esfuerzo desarrollado en esta dirección.

Las perovskitas de manganeso con valencia mixta tienen por fórmula general $\mathrm{A}_{1-\mathrm{x}} \mathrm{B}_{\mathrm{x}} \mathrm{MnO}_{3^{\prime}}$ donde $\mathrm{A}$ es una tierra rara del grupo de los lantánidos $\left(\mathrm{La}^{3+}, \mathrm{Pr}^{3+}, \mathrm{Nd}^{3+}\right.$, etc. $)$ y $\mathrm{B}$ es generalmente un alcalino-terreo (como el $\mathrm{Ca}^{2+}, \mathrm{Sr}^{2+}$, etc.). La desigualdad de estas valencias provoca la coexistencia de iones $\mathrm{Mn}^{3+} / \mathrm{Mn}^{4+}$ con el objeto de mantener la neutralidad de la carga. La interacción entre estas especies, llamada interacción de doble intercambio (DI) ya fue estudiada hace 40 años (5) y explica el comportamiento eléctrico y magnético de estos óxidos, al menos cualitativamente. Según esta teoría, los electrones móviles de los orbitales $\mathrm{e}_{\mathrm{g}}$ del $\mathrm{Mn}^{3+}$ pueden saltar a los estados libres en el $\mathrm{Mn}^{4+}$, pero conservando su spin debido al fuerte acoplamiento de Hund con los electrones $\mathrm{t}_{2 \mathrm{~g}}$; de esta manera, el salto electrónico se ve favorecido cuando los spines de iones contiguos están alineados paralelamente. Por lo tanto, para ciertos grados de dopaje típicos $(0.2<\mathrm{x}<0.5)$, la conducción eléctrica se encuentra asociada al ferromagnetismo (6). Este hecho provoca la simultaneidad de la transición ferromagnética-paramagnética con la metal-aislante $\left(\mathrm{T}_{\mathrm{C}} \approx \mathrm{T}_{\mathrm{M}-\mathrm{A}}\right)$. Sin embargo, esta teoría no es suficiente para predecir los elevados valores de magnetorresistencia que se obtienen experimentalmente en un entorno de la transición de fase. Para ello, en los últimos años se han desarrollado propuestas más complejas $(7,8)$, que toman como base el doble intercambio, pero que añaden nuevos elementos que proporcionan una mayor fidelidad a los datos experimentales, básicamente una fuerte interacción spinred. Esta interacción podría estar asociada a la distorsión Jahn-Teller que sufre el octaedro $\mathrm{Mn}^{3+} \mathrm{O}_{6^{\prime}}$ y que se propaga a lo largo de toda la red gracias a los electrones itinerantes. Este efecto da lugar a una distorsión estructural, que pasa de O-ortorrómbica $(\mathrm{a}<\mathrm{c} / \sqrt{2}<\mathrm{b})$ a $\mathrm{O}^{\prime}$-ortorrómbica $(\mathrm{c} / \sqrt{ } 2<\mathrm{a}<\mathrm{b})$.

No obstante, han aparecido nuevos fenómenos que se han demostrado quizás aún más interesantes que la propia MRC; uno de ellos es el del transporte intergranular (9). Conjuntamente con la magnetorresistencia intrínseca de estos materiales, aparece otro tipo de magnetorresistencia, fuera de la transición de fase, por el paso de los electrones a través de las fronteras de grano en muestras policristalinas, bien sean películas delgadas o materiales cerámicos. Por lo tanto, los dos tipos de magnetorresistencia se muestran simultáneamente y compiten en las muestras granulares.

El origen de la magnetorresistencia intergranular (MRI) se encuentra en que en un proceso de efecto túnel entre dos gra- 
nos, si el electrón conserva su spin aparece una energía magnética de acoplamiento adicional cuando los momentos magnéticos de los granos adyacentes no se encuentran paralelos (10). De esta forma surge un mecanismo muy sensible al efecto del campo magnético que tiene especial relevancia cuando el grado de polarización de spin de los electrones de conducción es muy alto, tal y como ocurre en las perovskitas de manganeso (11). Debido a la influencia de las fronteras en la MRI, es lógico pensar que al reducir el tamaño de grano, este efecto se vea multiplicado.

\section{PROCEDIMIENTO EXPERIMENTAL}

Para realizar este trabajo se prepararon muestras de $\mathrm{La}_{0.67} \mathrm{Ca}_{0.33} \mathrm{MnO}_{3}$ por los métodos cerámico y sol-gel. Las muestras cerámicas se prepararon a partir de óxidos de alta pureza $\left(\mathrm{CaO}, \mathrm{La}_{2} \mathrm{O}_{3}, \mathrm{MnO}\right.$ y $\left.\mathrm{MnO}_{2}\right)$ por el método convencional de tratamientos térmicos y moliendas sucesivas. En este caso, el tratamiento final fue de 100 horas a $1300^{\circ} \mathrm{C}$. Las muestras de tamaño de grano nanométrico se prepararon por el método sol-gel, que ha sido descrito en detalle por Vázquez-Vázquez et al. (12). Este método consiste básicamente en la preparación de una solución acuosa de $\mathrm{La}\left(\mathrm{NO}_{3}\right)_{2} \cdot 6 \mathrm{H}_{2} \mathrm{O}, \mathrm{Mn}\left(\mathrm{NO}_{3}\right)_{2} \cdot 6 \mathrm{H}_{2} \mathrm{O}, \mathrm{Ca}\left(\mathrm{NO}_{3}\right)_{3} \cdot 4 \mathrm{H}_{2} \mathrm{O}$ en las proporciones estequiométricas deseadas y urea, que se emplea como agente gelificante. Esta solución se evapora lentamente hasta que se forma un gel; posteriormente el gel se descompone calentándolo a $250^{\circ} \mathrm{C}$ por un tiempo de 3 horas, lo que nos da como resultado una ceniza precursora de las fases que queremos obtener. El precursor se calienta a temperaturas entre $700^{\circ} \mathrm{C}$ y $1100^{\circ} \mathrm{C}$ por 6 horas para obtener muestras de diferentes tamaños de grano.

Los materiales obtenidos se caracterizaron estructuralmente mediante difracción de rayos X (Phillips modelo PW-1710, radiación $\mathrm{Cu} \mathrm{K}_{\alpha}$ ), ajustando los patrones obtenidos mediante el método de Rietveld. Para estudiar la morfología y el tamaño de partículas se empleó un microscopio electrónico de barrido (JEOL modelo 2010, 200kV). El contenido de oxígeno de las muestras se determinó por medio de yodimetrías.

Las propiedades de transporte se determinaron por el método de cuatro puntas en un intervalo de temperaturas desde $4 \mathrm{~K}$ hasta $300 \mathrm{~K}$ y en campos de hasta $50 \mathrm{kOe}$. La magnetización se midió en el mismo intervalo de temperaturas por medio de un magnetómetro SQUID Quantum Design y un magnetómetro de muestra vibrante (DMS 990). La magnetorresistencia se define de la manera usual como $\% \mathrm{MR}=100 x(\rho(\mathrm{H}=0)-\rho(\mathrm{H}) / \rho(\mathrm{H}=0))$.

\section{RESULTADOS}

\subsection{Caracterización de las muestras}

Las muestras obtenidas por los dos métodos empleados resultaron ser perfectamente cristalinas y sin presencia alguna de fases secundarias, tal y como se observó a través del análisis por medio del método de Rietveld de los patrones de difracción de rayos X. Los tamaños de grano (D), obtenidos por microscopía, se muestran en la Tabla I. Como se observa, podemos obtener una gran variación en este parámetro (hasta casi 3 órdenes de magnitud) lo que nos lleva a observar múltiples efectos en nuestro compuesto. La distribución de tamaños tiene una desviación aproximada del 15\% en las muestras realizadas por el método sol-gel, mientras que la forma de las partículas resultó ser ligeramente alongada, apartándose ligeramente de la esfericidad.

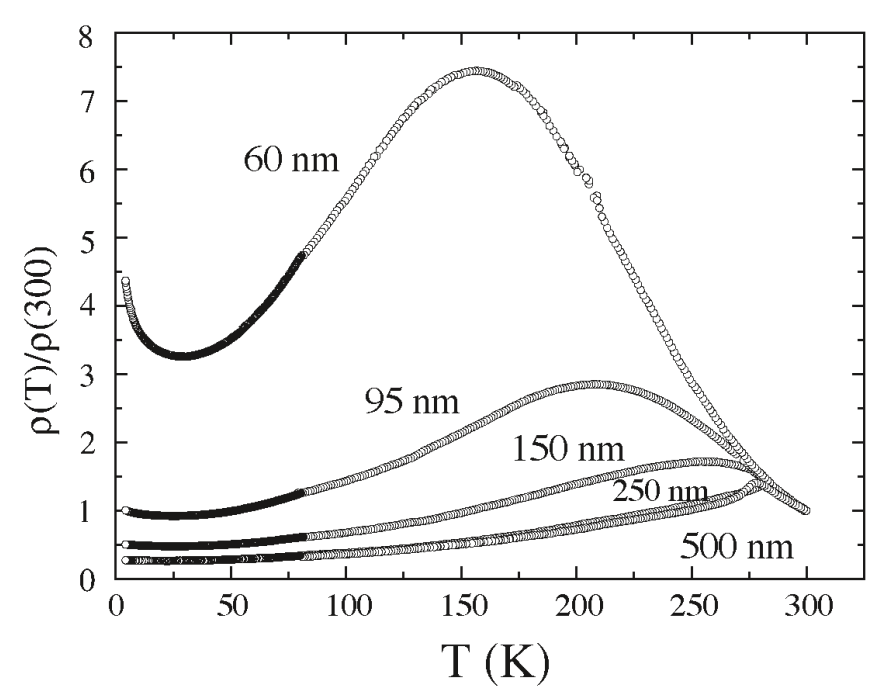

Figura 1. Resistividad reducida frente a la temperatura para muestras nanocristalinas con tamaños de grano comprendidos entre 60 $\mathrm{nm}$ y $500 \mathrm{~nm}$.

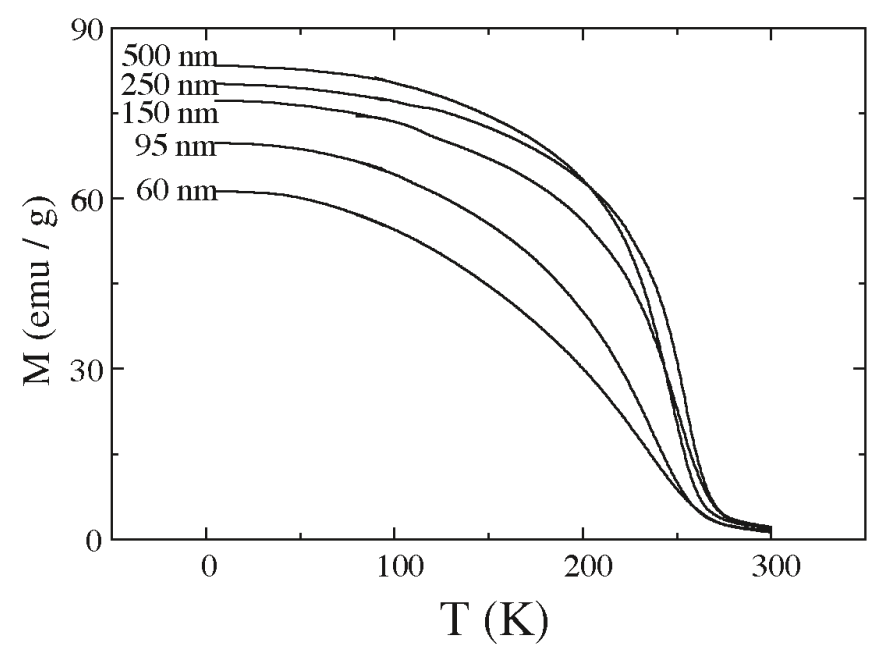

Figura 2. Dependencia con la temperatura de la magnetización medida a 5 kOe para las muestras nanocristalinas estudiadas.

TABLA I. VARIACIÓN DEL TAMAÑO DE PARTíCUlA SEGÚN EL MÉTODO DE OBTENCIÓN EMPLEADO Y LA TEMPERATURA DE SINTERIZACIÓN.

\begin{tabular}{lcc}
\hline MÉTODO & $\begin{array}{c}\text { TEMPERATURA } \\
\text { SINTERIZACIÓN }\end{array}$ & $\begin{array}{c}\text { TAMAÑO } \\
\text { PARTÍCULA }\end{array}$ \\
\hline Sol-Gel & $700^{\circ} \mathrm{C}(6 \mathrm{~h})$ & $60 \pm 9 \mathrm{~nm}$ \\
Sol-Gel & $800^{\circ} \mathrm{C}(6 \mathrm{~h})$ & $95 \pm 14 \mathrm{~nm}$ \\
Sol-Gel & $900^{\circ} \mathrm{C}(6 \mathrm{~h})$ & $150 \pm 21 \mathrm{~nm}$ \\
Sol-Gel & $1000^{\circ} \mathrm{C}(6 \mathrm{~h})$ & $250 \pm 38 \mathrm{~nm}$ \\
Sol-Gel & $1100^{\circ} \mathrm{C}(6 \mathrm{~h})$ & $500 \pm 100 \mathrm{~nm}$ \\
Cerámico & $1300^{\circ} \mathrm{C}(100 \mathrm{~h})$ & $\approx 20 \mu \mathrm{m}$ \\
\hline
\end{tabular}




\subsection{Propiedades de magnéticas y de transporte}

Podemos estudiar simultáneamente las propiedades de transporte y magnéticas de las diferentes muestras, ya que, como comentamos, están muy relacionadas. En primer lugar hemos representado la resistividad reducida para las muestras con diferentes tamaños de grano (figura 1). Como se observa, cuando el tamaño de partícula se reduce, el valor de la resistividad aumenta, mientras que el valor de la temperatura de transición metal-aislante $\left(\mathrm{T}_{\mathrm{M}-\mathrm{A}}\right)$ disminuye. Este comportamiento ha sido explicado teniendo en cuenta la influencia en la resistividad total de las fronteras entre granos, que aumenta al disminuir el tamaño de los mismos (13), y por otra parte, en la no estequiometría de oxígeno que presentan las muestras tratadas a bajas temperaturas (14). Este último es uno de los mayores problemas que aparecen en este tipo de compuestos pues hasta hace poco tiempo no se sabía exactamente cuál era su influencia en las propiedades de transporte finales, y que consiste, como se observa en la figura 2, en que la temperatura de Curie $\left(\mathrm{T}_{\mathrm{C}}\right)$, permanece prácticamente constante mientras vimos como $\mathrm{T}_{\mathrm{M}-\mathrm{A}}$ se reduce. Aparece por lo tanto una zona de comportamiento aislante y ferromagnético, que se puede explicar en función de zonas ferromagnéticas, suficientemente grandes como para dar una respuesta magnética, pero aisladas unas de otras, por lo que se interrumpe la conducción (14).

Por otra parte, podemos apreciar claramente un aumento de resistividad a bajas temperaturas $(\mathrm{T}<30 \mathrm{~K})$ para las muestras con un tamaño de grano lo suficientemente pequeño $(\mathrm{D}<150 \mathrm{~nm})$. Sin embargo, las propiedades magnéticas no presentan ninguna anomalía en esta zona (ver figura 2). Estudios teóricos sugieren la posibilidad de la existencia de Bloqueo de Coulomb entre granos que tengan un tamaño adecuado $(15,16)$. La aparición de una pequeña barrera de origen electrostático entre los granos, que se añade a la barrera de tipo magnético y estructural ya conocida, sería la que provocaría el aumento de resistencia observado a muy bajas temperaturas.

Esta hipótesis se ve reafirmada por los ajustes que se han realizado de la resistividad a bajas temperaturas en las muestras de grano más pequeño a la expresión teórica $\rho(T)=A \exp (\sqrt{C / T})$, tal y como se puede observar en la figura $\rho(T)=A \exp (\sqrt{C / T})_{3}^{\prime}$. En esta fórmula, la constante $C$ es proporcional a la energía electrostática que aparece entre los granos, y que aumenta de valor conforme se reduce el tamaño de partícula, esto es, cuando la superficie tiene más importancia (15). Estas conclusiones se podrían confirmar con medidas de impedancia compleja, de manera que los resultados nos permitan diferenciar más claramente las contribuciones del material masivo y de los bordes de grano.

Por otra parte, la magnetorresistencia es uno de los parámetros que varían de una manera más radical cuando se varía el tamaño de partícula. Como ya comentamos, cerca de la transición metal-aislante, los materiales monocristalinos o los compuestos cerámicos presentan un pico de magnetorresistencia intrínseca al material. Sin embargo, hemos observado como al reducir el tamaño de grano, este pico de magnetorresistencia desaparece progresivamente (fig. 4). La explicación a este comportamiento se ha buscado en teorías que relacionan la magnetorresistencia en las perovskitas de manganeso con la presencia de paredes de dominio (17). En las muestras con granos más pequeños, éstos son aproximadamente monodominios, por lo que la ausencia de desordenes magnéticos en forma de paredes en el interior de las partículas puede llevar asociada la

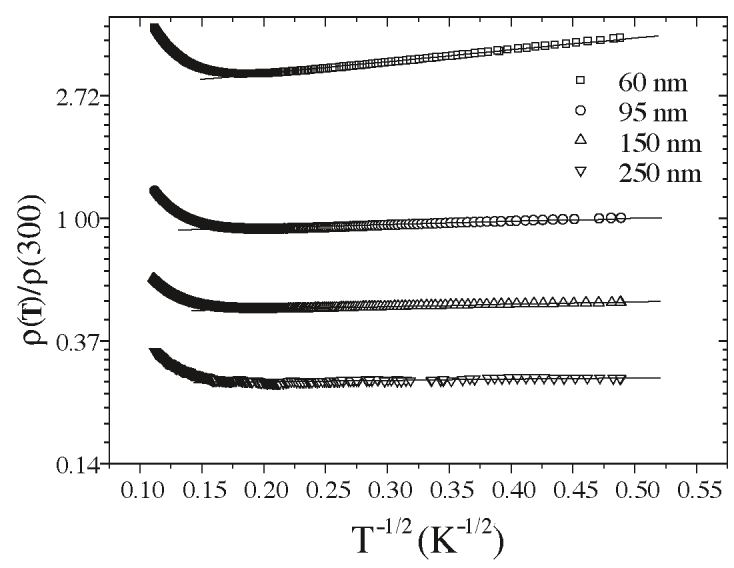

Figura 3. Ajustes de la resistividad reducida a bajas temperaturas según la expresión existencia de una $\rho(T)=A \exp (\sqrt{C / T})$ barrera de origen electrostático entre los granos.

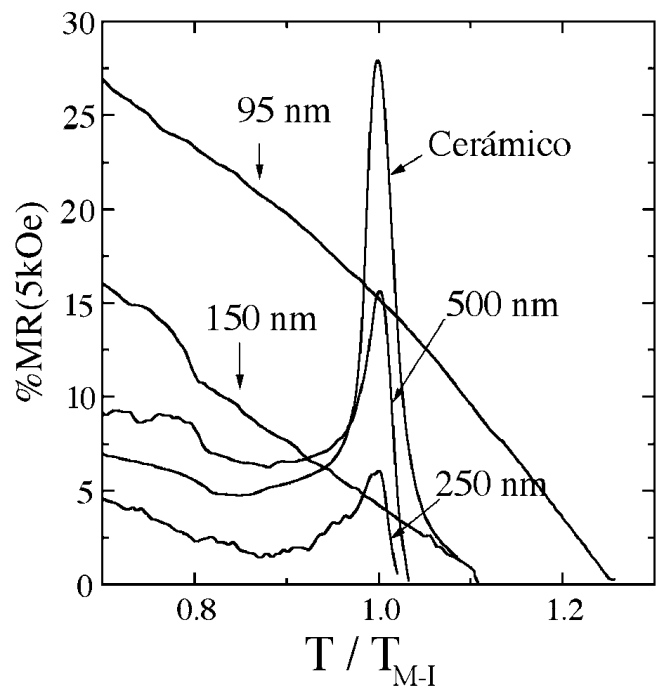

Figura 4. Variación del porcentaje de magnetorresistencia frente a la temperatura reducida. Se observa claramente la claramente la destrucción del pico de MRC en la transición de fase magnética al reducirse el tamaño de partícula.

destrucción de la MRC cerca de la transición de fase (18).

Simultáneamente a la destrucción de la magnetorresistencia colosal, se aprecia un aumento claro de la magnetorresistencia intergranular proporcional a la reducción del tamaño de partícula (fig. 4). Al reducirse el tamaño, aumenta la relación superficie-volumen, por lo que los efectos de frontera se ven ampliados en una proporción $1 / \mathrm{D}$, donde $\mathrm{D}$ es el diámetro de las partículas. Esta conclusión está en clara concordancia tanto con desarrollos teóricos (10) como con resultados experimentales obtenidos por otros grupos $(9,13)$. Sin embargo, a temperaturas más bajas, la magnetorresistencia parece no estar tan afectada por la gran variación en el tamaño de partícula, tal y como podemos notar en la figura 5. El efecto se ve atenuado al reducirse todavía más la temperatura, especialmente a campos altos (40 kOe), tal y como podemos ver también en la figura 6 .

La mayor parte de la contribución a la MRI se realiza a campos bajos $(\mathrm{H}<5 \mathrm{kOe})$. Este efecto se puede ver de una forma más clara en la figura 6, en la que se muestra las isotermas de magnetorresistencia para la muestra de $95 \mathrm{~nm}$ de tamaño de partí- 


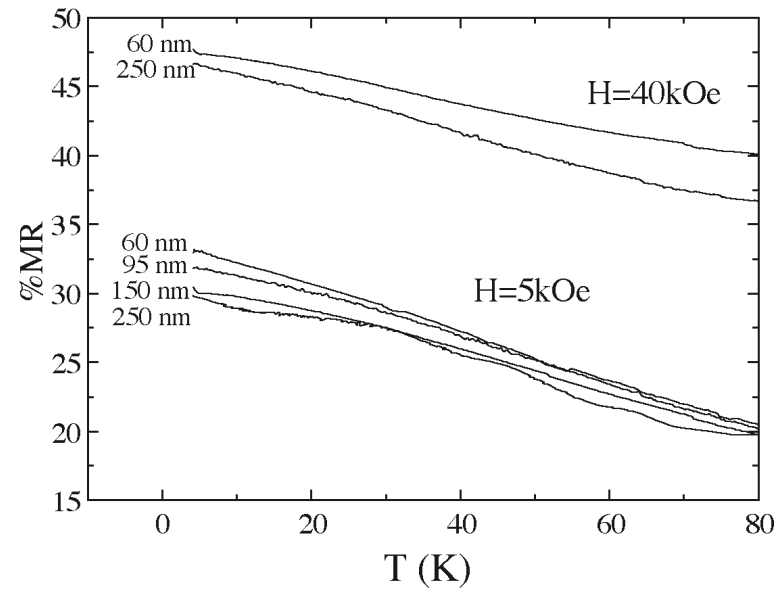

Figura 5. Variación del porcentaje de magnetorresistencia a bajas temperaturas para diversas muestras nanocristalinas. Se presentan los resultados a bajos $(\mathrm{H}=5 \mathrm{kOe})$ y altos $(\mathrm{H}=40 \mathrm{kOe})$ para comparar los resultados.

cula. Hay un descenso muy brusco de la resistencia hasta unos $5 \mathrm{kOe}$, y para campos más altos, una zona aproximadamente lineal para la que no se observa saturación en el límite experimental de nuestro equipamiento. Este es un efecto interesante, pues la mayor parte de las aplicaciones tecnológicas demandan respuestas apreciables y aproximadamente lineales a bajos campos.

\section{CONCLUSIONES}

En este trabajo hemos estudiado sistemáticamente los diferentes efectos que se producen en la perovskita $\mathrm{La}_{0.67} \mathrm{Ca}_{0.33} \mathrm{MnO}_{3}$ al reducirse el tamaño de grano. Por una parte hemos observado cómo la resistividad global de las muestras aumenta significativamente al mismo tiempo que se reduce la temperatura de transición metal-aislante. Este efecto se ve producido en gran medida por la presencia de vacantes de oxígeno en las muestras sinterizadas a bajas temperaturas. Simultáneamente, aparece una zona de comportamiento de tipo "semiconductor" a bajas temperaturas que puede ser explicada en términos de la aparición de una barrera de origen coulombiano entre granos lo suficientemente pequeños. Por último, debemos destacar los efectos que sobre la magnetorresistencia tiene la reducción del tamaño de partícula. En la zona de la transición metal-aislante, la MRC se destruye al reducirse el tamaño de partícula, desapareciendo para $\mathrm{D} \leq 150 \mathrm{~nm}$. Al mismo tiempo, la magnetorresistencia intergranular se ve favorecida por esta disminución del tamaño de grano.

\section{AGRADECIMIENTOS}

Dos de los autores (L.E.H. y F.R.) desean agradecer la concesión de una beca F.P.I. al M.E.C.. Este trabajo está financiado por el proyecto de la CICYT MAT98-0416.

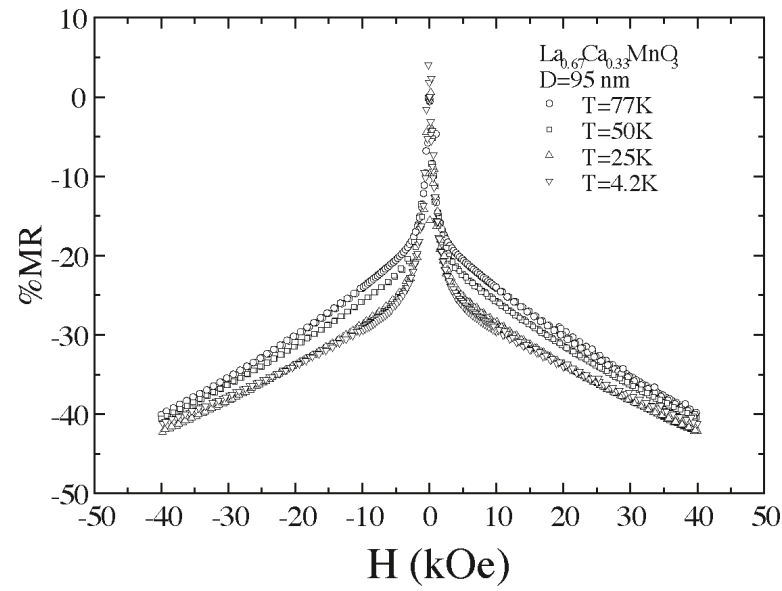

Figura 6. Porcentaje de magnetorresistencia frente al campo magnético a varias temperaturas para la muestra con tamaño de grano de $95 \mathrm{~nm}$. Se puede distinguir la zona de bajo campo y la zona de alto campo para varias temperaturas.

\section{BIBLIOGRAFÍA}

1. M.N. Baibich, J.M. Broto, A. Fert, F. Nguyen van Dau, F. Petroff, P. Etienne, G. Creuzet, A. Friedich, J. Chazelas, "Giant magnetoresistance of (001)Fe/(001)Cr magnetic superlattices", Phys. Rev. Lett. 61, 2472-2475 (1988).

2. A. Berkowitz, A.P. Young, J.R. Mitchell, S. Zhang, M.J. Carey, F.E. Spada, F.T. Parker, A. Hutten, G. Thomas, "Giant magnetoresistance in heterogeneous Cu-Co alloys", Phys. Rev. Lett. 68, 3745-3748 (1992).

3. R. von Helmolt, J. Wecker, B. Holzapfel, L. Schultz, K. Samwer, "Giant negative magnetoresistance in perovskitelike $\mathrm{La}_{2 / 3} \mathrm{Ba}_{1 / 3} \mathrm{MnO}_{x}$ ferromagnetic films" Phys. Rev. Lett. 71, 2331-2333 (1993).

4. S. Jin, T.H. Tiefel, M. McCormack, R.A. Fastnacht, R. Ramesh, L.H. Chen, "Thousandfold change in resistivity in magnetoresistive La-Ca-Mn-O films", Science 264, 412-415 (1994).

5. P.G. de Gennes, "Effects of double exchange in magnetic crystals", Phys. Rev. 118, 141-154 (1960).

6. P. Schiffer, A.P. Ramirez, W. Bao, S-W. Cheong, “Low temperature magnetoresistance and the magnetic phase diagram of $\mathrm{La}_{1-\mathrm{x}} \mathrm{Ca}_{\mathrm{x}} \mathrm{MnO}_{3}{ }^{\prime}$, Phys. Rev. Lett. 75, 3336-3339 (1995).

7. H. Röder, J. Zhang, A.R. Bishop, "Lattice effects in the colossal magnetoresistive manganites", Phys. Rev. Lett. 76, 1356-1359 (1996).

8. A.J. Millis, B. Shraiman, R. Mueller, "Dynamic Jahn-Teller effect and colossal magnetoresistance in $\mathrm{La}_{1-\mathrm{x}} \mathrm{Sr}_{x} \mathrm{MnO}_{3}$ ", Phys. Rev. Lett. 77, 175-178 (1996).

9. H.Y. Hwang, S-W. Cheong, N.P. Ong, B. Batlogg, "Spin-polarized tunneling in $\mathrm{La}_{2 / 3} \mathrm{Sr}_{1 / 3} \mathrm{MnO}_{3}$ ", Phys. Rev. Lett. 77, 2041-2044 (1996).

10. J.S. Helman, B. Abeles, "Tunneling of spin-polarized electrons and magnetoresistance in granular Ni films", Phys. Rev. Lett. 37, 1429-1432 (1976).

11. J.-H. Park, E. Vescovo, H.-J. Kim, C. Kwon, R. Ramesh, T. Venkatesan, “Direct evidence for a half-metallic ferromagnet", Nature 392, 794-796 (1998).

12. C. Vázquez-Vázquez, M.C. Blanco, M.A. López-Quintela, R.D. Sánchez, J. Rivas, S.B. Oseroff, "Characterization of $\mathrm{La}_{0.67} \mathrm{Ca}_{0.33} \mathrm{MnO}_{3 \pm \mathrm{d}}$ prepared by the sol-gel route", J. Mater. Chem 8, 991-1002 (1998).

13. A. Gupta, G.Q. Gong, G. Xiao, P.R. Duncombe, P. Lecoeur, P. Trouilloud, Y.Y. Wang, V.P. Dravid, J.Z. Sun, “Grain-boundary effects on the magnetoresistance properties of the perovskite manganite films", Phys. Rev. B 54, 15629-15632 (1996).

14. L.E. Hueso, F. Rivadulla, R.D. Sánchez, D. Caeiro, C. Jardón, C. VázquezVázquez, J. Rivas, M.A. López-Quintela, “Influence of the grain-size and oxygen stoichiometry on the magnetic and transport properties of polycrystalline $\mathrm{La}_{0.67} \mathrm{Ca}_{0.33} \mathrm{MnO}_{3 \pm d}$ perovskites", J. Mag. Mag. Mat. 189, 321-328 (1998).

15. Ping Sheng, B. Abeles, Y Arie, "Hopping conductivity in granular metals", Phys. Rev. Lett. 31, 44-47 (1973).

16. F. Guinea, "Spin-flip scattering in magnetic junctions", Phys. Rev. B 58, 9212-9216 (1998).

17. S. Zhang, Z. Yang, "On the origin of magnetoresistance in ferromagnetic perovskite structures", J. Appl. Phys. 79, 7398-7400 (1996).

18. L.E. Hueso, J. Rivas, F. Rivadulla, M.A. López-Quintela, "Tuning of colossal magnetoresistance via grain size change in $\mathrm{La}_{0.67} \mathrm{Ca}_{0.33} \mathrm{MnO}_{3}$ ", J. Appl. Phys. 86, 3881-3884 (1999). 